\title{
The nature of cell-cycle checkpoints: facts and fallacies
}

\author{
Alexey Khodjakov and Conly L Rieder \\ Address: Wadsworth Center, PO Box 509, Albany, NY 12201-0509, USA. \\ Correspondence: Alexey Khodjakov. Email: khodj@wadsworth.org; Conly L Rieder. Email: rieder@wadsworth.org
}

\begin{abstract}
The concept of checkpoint controls revolutionized our understanding of the cell cycle. Here we revisit the defining features of checkpoints and argue that failure to properly appreciate the concept is leading to misinterpretation of experimental results. We illustrate, using the mitotic checkpoint, problems that can arise from a failure to respect strict definitions and precise terminology.
\end{abstract}

"Cell biology is not a notoriously self-critical field. We cell biologists are not reticent about announcing breakthroughs and making promises of imminent revolutions. However, one cannot summarize the history of research and thought about mitosis as a progress from primitive glimmerings to modern revelations. Nothing we have learned about mitosis since it was discovered a century ago is as dazzling as the discovery itself" [1].

Without doubt, each scientific generation gathers more information about mitosis than its predecessors. But despite stunning advances in imaging that allow many of the intimate details of spindle assembly and chromosome behavior to be visualized, and concurrent strides in molecular genetics and biochemistry that have identified a plethora of molecules and interactions directly or indirectly required for proper mitosis, major conceptual advances are, as opined by Daniel Mazia in the quotation above, rare. Yet, we believe that the concept of cell-cycle checkpoint controls articulated in the late 2oth century by Leland Hartwell (for which he shared the Nobel Prize in 2001) was a breakthrough to rival the discovery of mitosis itself.

Hartwell's idea departed from the traditional view that stage-to-stage progress through the cell cycle occurred whenever there were sufficient means to move forward. Instead, he argued that progression is actively controlled by external mechanisms that are not themselves intrinsic to the process. Checkpoints guard critical cell-cycle transitions by ensuring that the previous phase is complete and error-free before the cell is allowed to move forward. However, since its introduction 20 years ago the checkpoint concept has been re-tailored to suit a variety of views, many of which are based on misleading terminology and misconceptions. For example, delays in mitosis are often ascribed to 'activation' of the mitotic checkpoint, a descriptor that fails to recognize that the checkpoint by definition is active as the cell starts mitosis. Conversely, the completion of mitosis in the presence of misaligned chromosomes is often automatically interpreted to indicate a defective checkpoint, even though in the absence of critical testing alternative interpretations are equally likely. In this article we define the critical characteristics of checkpoints and illustrate how confusion generated by the inconsistent use of terminology may impede progress by fostering claims that mean very different things to different researchers. We will illustrate our points with examples from the checkpoint that controls progression through mitosis (Figure 1).

Checkpoints are not essential (in happy cells) The existence of critical 'triggers' or 'points of no return' at key cell-cycle transitions was postulated by Mazia as early as 1961 [2], although, as he later acknowledged in 1987, the concept as originally formulated proved non-productive. The problem was that the triggers were envisaged to be essential internal components of the molecular cascades that drive the cell cycle. Checkpoints, by contrast, are external control mechanisms that are not required for forward progression [3]. Thus, a fundamental feature of a checkpoint is that its activities are not manifested under conditions in which the potential for errors is minimal: only when conditions become stressful and errors are likely to occur do checkpoints become essential survival tools. This criterion formed the basis of early screens to identify mitotic checkpoint components in yeast [4]. The name of three well known mitotic checkpoint proteins, Mad1-3, comes from the acronym 'Mitotic Arrest Deficient', reflecting the fact that Mad mutants progress through mitosis with similar kinetics whether or not the spindle is present (and thus in the presence of unattached kinetochores, which normally arrest mitosis - see legend to Figure 1). In contrast, wild-type cells arrest in mitosis when spindle formation is inhibited with microtubule poisons. Under normal conditions, however, both wild-type and Mad-deficient cells or organisms with low chromosome number and efficient spindle assembly mechanisms (for example, yeast and Drosophila) grow equally well, which 


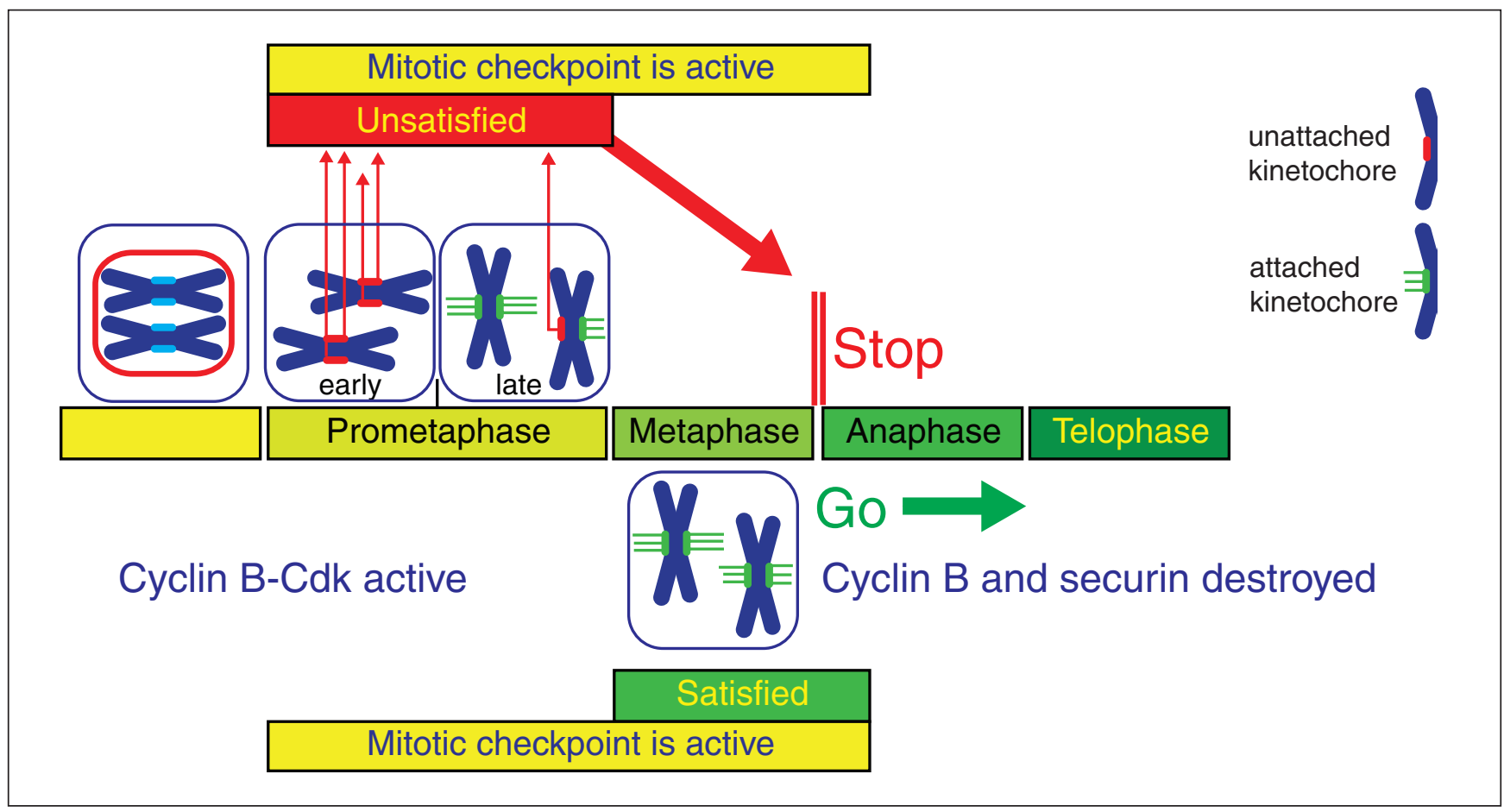

Figure 1

The operation of the mitotic checkpoint. The cell cycle is driven by cyclin-dependent kinases (CDKs), which are activated by binding to cyclins that are specific for the different phases of the cell cycle and determine the targets of the kinases. Exit from each phase of the cell cycle occurs on degradation of the bound cyclin. The CDK-cyclin complex that is required for entry into mitosis is CDK1-cyclin B, and cells are driven from G2 into mitosis by its sudden activation. Exit from mitosis at anaphase occurs on activation of the anaphase-promoting complex (APC), a large ubiquitin ligase that targets cyclin B for degradation. The securin that holds the mitotic chromosomes together at metaphase is also tagged for degradation by the APC. The mitotic checkpoint is an external monitoring system that by itself is not required for mitotic progression but detects the presence of chromosomes that are not attached to the mitotic spindle via their kinetochores and, in their presence, initiates a cascade that prohibits activation of the APC and thus chromosome separation and exit from mitosis. When the last kinetochore attaches to microtubules the checkpoint becomes satisfied, allowing APC activation and progress towards mitotic exit. However, even when satisfied, the checkpoint pathway continues to survey for unattached kinetochores, which, should they arise, readily re-impose the block.

reflects the fact that the mitotic checkpoint is not essential when the frequency of errors is naturally low.

Some argue that the function of the mitotic checkpoint in yeast and Drosophila is different from that in mammals, because in mammals inactivation of checkpoint genes is lethal even in the absence of other stresses. This argument is conceptually flawed, as the fate difference observed simply reflects differences in the speed of spindle assembly. Because of the stochastic nature of interaction between kinetochores and spindle microtubules, the presence of numerous chromosomes and/or centrosomes (spindle poles) greatly increases the time required for spindle assembly. Under this condition, unless mitotic exit is delayed by the checkpoint until all kinetochores have attached to the spindle the progeny will be aneuploid. For this reason, inactivating the mitotic checkpoint in mammals results in a rapid rise in aneuploidy and ultimately death [5]. A nice illustration of the interplay between the checkpoint, the kinetics of spindle assembly, and cell/organism viability comes from recent work in Drosophila that accumulate supernumerary centrosomes. Although this condition itself does not compromise viability, it slows the rate of spindle assembly. Predictably, eliminating the mitotic checkpoint by deleting Mad2, which has no effect on wild-type Drosophila, becomes lethal in flies with supernumerary centrosomes [6]. It is important to emphasize that in the latter case, cells are not 'killed by the checkpoint' as sometimes described in the literature. Instead, cells die because they ultimately become highly aneuploid in the absence of a functional checkpoint. The function of the mitotic checkpoint is to prevent premature mitotic exit - and nothing else.

The failure to distinguish true checkpoint proteins from those involved in the pathway targeted by the mitotic checkpoint is common, and usually results from too narrow a focus on molecular interactions without regard for the conceptual context. It is obvious that checkpoint proteins must interact not only with the structure or event 
being monitored (for example, an unattached kinetochore), but also with the pathway and structures whose activity is required to drive cell-cycle progression. This being the case, because the checkpoint itself is not required for forward progression, proteins whose mutations prolong mitosis can never be considered true checkpoint components.

For example, exit from mitosis requires the activation of a large ubiquitin ligase, termed the anaphase-promoting complex (APC) or cyclosome, which tags for destruction proteins that hold replicated chromosomes together or that keep the cell in mitosis (these include securin and cyclin B; Figure 1). The APC is activated by an activator protein, Cdc2O, and if Cdc2O is depleted, the cell arrests in mitosis. Despite the fact that Cdc2o interacts directly with bona fide checkpoint proteins (for example, Mad2), this phenotype clearly demonstrates that Cdc2O itself is not a checkpoint protein. Interaction between Cdc2O and checkpoint proteins is expected as the goal of the mitotic checkpoint is, biochemically, to prevent premature activation of the APC by sequestering Cdc20. Mistakenly considering Cdc2O or other proteins intrinsically required for forward mitotic progression to be directly involved in the checkpoint degrades the checkpoint concept back to Mazia's internal 'triggers'.

\section{Once the bird has flown it is too late to lock the cage}

Although checkpoint activities during mitosis are not apparent in the absence of persistent errors, this does not mean that the checkpoint is inactive, as implied by the alltoo-common claim that a condition or treatment suddenly 'activates' or 'triggers' the checkpoint. These are misleading oxymora: as the role of the checkpoint is to detect a problem, the monitoring mechanism (that is, the checkpoint) must be already active before the problem arises (before the bird escapes the cage). Like all checkpoints, the mitotic checkpoint is a constitutive pathway that is active at the start of spindle assembly.

Contrary to the views of some, the mitotic checkpoint is not 'turned off' once it is satisfied, but continues to remain functional. This is evident from the fact that treating cells with spindle poisons after they have initiated mitotic exit rapidly stops further cyclin B degradation and progress towards anaphase [7]. Thus, up to a point, reappearance of the condition monitored by the checkpoint reinstates the block. That point at which the checkpoint becomes truly inactivated marks a point of no return after which progression to the next stage of the cell cycle can no longer be stopped.

\section{Two ways to cross the border: get a visa or incapacitate the guard}

Another fundamental property of a checkpoint is that there are always two ways to progress past it. One is to satisfy it by eliminating the condition it monitors. The other is to abrogate the checkpoint itself. (Space considerations preclude a discussion of checkpoint adaptation in yeast or slippage in mammals, which, as biological controls are not 100\% efficient, allow some cells to ultimately escape mitosis after a prolonged block.) A failure to distinguish between satisfying and abrogating the mitotic checkpoint frequently leads to erroneous interpretations of checkpoint phenotypes. There is an unwarranted tendency to conclude that the checkpoint is defective whenever progression proceeds in the presence of maloriented or mis-positioned chromosomes.

The chromosomal instability (CIN) story nicely illustrates this point. For years it was assumed that the individual chromosome mis-segregation phenotype of CIN cells resulted from a defective or weakened mitotic checkpoint. However, recent live-cell studies have revealed that the mitotic checkpoint in most CIN cells is perfectly normal [8]. Instead, the so-called checkpoint-deficient phenotype in CIN results from abnormal spindle microtubule dynamics, and/or problems in the constitutive mechanism responsible for correcting chromosome attachment errors that is not part of the checkpoint cascade [9]. These problems allow the checkpoint to ultimately be satisfied under conditions in which chromosome segregation may not be normal.

As noted by Hartwell and Weinert [3], "the existence of a control mechanism is suggested when one finds chemicals, mutants, or other conditions that ... permit a late event to occur even when an early, normally prerequisite event is prevented". Because this relief-of-dependence criterion is a hallmark of a checkpoint control, one can conclude that the mitotic checkpoint is really defunct in a cell only if it fails to delay in mitosis under conditions that are known to prohibit its satisfaction. In practice, the best test for a deficient mitotic checkpoint is the extent that cells are delayed in mitosis in the absence of spindle microtubules. (Note that the use of drugs or conditions that simply perturb microtubule dynamics, for example, Taxol or low concentrations of nocodazole, are not informative about the mitotic checkpoint because they still allow it to be satisfied, sometimes very rapidly [10].)

\section{Just how many mitotic checkpoints are there?}

Clearly, several different conditions must be met during mitosis to ensure that the replicated chromosomes are equally distributed into daughter cells. Thus, one can envisage multiple checkpoints (or multiple branches of one checkpoint), each detecting one of these conditions. Alternatively, a range of abnormalities may ultimately distil into a single condition detected by just one checkpoint. These are very different possibilities: the former implies the existence of complex multiple independent feedback loops while the latter relies on a single master guard.

From laser ablation studies it is clear that a single unattached kinetochore prevents satisfaction of the mitotic 
checkpoint [11]. It is similarly evident from solid biochemical and genetic evidence that generation of the "wait anaphase' checkpoint signal involves proteins like Mad2 that are present on unattached but not on attached kinetochores. Thus, there is no doubt that the problem detected by the mitotic checkpoint is the presence of kinetochores that are not attached to spindle microtubules. Given this, the question becomes whether there are other conditions that delay progression through mitosis that are independent of unattached kinetochores.

A prerequisite for equal chromosome segregation is that all chromosomes must acquire an amphitelic attachment to the spindle before the onset of anaphase: that is, one sister kinetochore must become attached to one spindle pole and the other to the opposing pole. However, during the normal course of spindle assembly chromosomes can acquire erroneous attachments: in some, both sister kinetochores become attached to the same pole (syntelic attachment), while in others a single kinetochore becomes simultaneously connected to both poles (merotelic attachment) (Figure 2). It makes intuitive sense to delay mitotic exit until these errors are corrected. However, a key nonintuitive, and thus often overlooked, fact is that such a delay is needed only if the mechanism for correcting such errors is slow. Just as the mitotic checkpoint is not essential in happy cells (see above), swift and efficient intrinsic correction mechanisms make the presence of an additional checkpoint pathway that detects improper kinetochore attachments unnecessary.

It is important to emphasize that correction of a syntelic attachment involves the separation of one kinetochore from its associated microtubule bundle. This results in an unattached kinetochore (a monotelic chromosome), which inevitably prevents satisfaction of the mitotic checkpoint (Figure 2). This readily explains why, even though syntelic attachments are not detected by the checkpoint, conditions that promote their formation prolong mitosis [12]. Indeed, when the error-correction mechanism is inhibited, for example, by knocking down kinesin 13 (a microtubule depolymerase), cells containing syntelic chromosomes rapidly satisfy the mitotic checkpoint because unattached kinetochores can no longer be generated. On the other hand, correction of the other type of erroneous attachments, merotelic, does not generate unattached kinetochores - which is why the presence of merotelic attachments does not delay cells in mitosis. In summary, there is no direct evidence that the mitotic checkpoint detects any problem or condition other than the presence of unattached kinetochores, not even chromosome mis-positioning or erroneous kinetochore attachments.

This being the case, what of the many claims that in addition to the kinetochore-based mitotic checkpoint, progress through mitosis is also controlled by various other

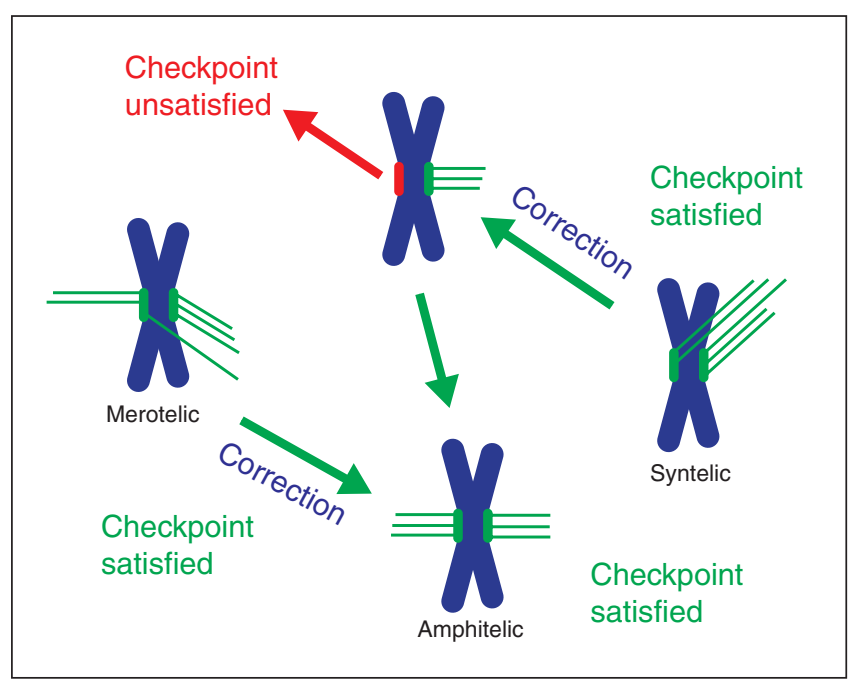

Figure 2

Unattached kinetochores occur in the course of correction of incorrectly attached chromosomes. Although the mitotic checkpoint cannot directly distinguish normal from erroneous kinetochore attachments, correction of the latter can result in the production of an unattached kinetochore detected by the checkpoint. See text for details.

pathways that respond to everything from the status of p53 or p38 to the integrity of the chromosomes and DNA catenation? First off, some of these claims fail to consider that multiple conditions, for example, anything that induces DNA damage, can make it difficult for one or more kinetochores to establish stable connections to the spindle. Thus, while a particular treatment or condition may indeed delay cells in mitosis, until the delay is convincingly demonstrated to be independent of the mitotic checkpoint there is no justification for claiming the presence of an additional checkpoint during mitosis (especially in mammals).

\section{'All that glisters is not gold'}

Confusion about the definition of a checkpoint, or what the mitotic checkpoint monitors, is one cause of unsubstantiated claims that progress through mitosis is regulated by checkpoint pathways other than the one that detects unattached kinetochores. Another is the use of widely different definitions for the same term in different biological fields. For example, a search of PubMed currently yields 3,505 papers under the key phrase 'mitotic progression'. The problem here is that a large fraction use the term to mean progress through the 'mitotic cell cycle' (old terminology for the cell cycle), while others consider it to mean (as we do) progression through mitosis. This being the case, claims that a particular pathway is needed for or involved in 'mitotic progression' often simply mean that it is in fact required for progression through G2 and not mitosis. A larger source of confusion arises from widespread use of the term 'G2/M', which originally came into 
use because fluorescence-activated cell sorting (FACS) cannot distinguish $4 N$ G2 cells from $4 N$ mitotic cells (or even $4 N \mathrm{G} 1$ cells that failed to segregate chromosomes). At this time there are 5,729 papers that deal with the 'G2/M cell', which obviously is an oxymoron as a cell cannot be in two different phases of the cell cycle simultaneously. Similarly, papers that focus on a 'G2/M arrest' $(2,437$ papers) or a 'G2/M checkpoint' (704 papers) should be treated simply as an admission of technical limitations that do not allow the authors to determine whether cells are in G2 or in mitosis. In spite of this major limitation, the term 'G2/M phase' (4,105 papers) is now commonly used as a bona fide stage (phase) of the cell cycle without considering the original meaning of the term. Just as there is no such thing as a G2/M cell or a G2/M phase, there is no such thing as a G2/M checkpoint. Rather, there are checkpoints that control progression through G2 and, as we have argued, there is a single checkpoint that controls progress through mitosis, but there is no clear evidence for any checkpoint that controls progression through both G2 and mitosis.

Few would disagree that scientific advances rest on the ability of the scientists involved to communicate clearly. An equally important part of scientific cognition is the ability to place individual findings into the larger context of previous and concurrent studies. This requires an in-depth understanding of the critical concepts that define the field. Failure to respect these concepts and the use of imprecise terminology divert attention from the real issues and may mask the limitations of individual experimental approaches and impede productive scientific communication.

\section{Acknowledgements}

We thank Tim Hunt for encouraging us to express these views. Research in our laboratories is supported by National Institutes of Health grants GM 59363 (to AK) and GM 40198 (to CLR). We apologize to our colleagues whose work was not properly cited in this opinion due to format limitations.

\section{References}

1. Mazia D: The chromosome cycle and the centrosome cycle in the mitotic cycle. Int Rev Cytol 1987, 100:49-92.

2. Mazia D: Mitosis and the physiology of cell division. In Meiosis and Mitosis. Edited by Brachet J, Mirsky AE. New York: Academic Press; 1961:77-412.

3. Hartwell LH, Weinert TA: Checkpoints: controls that ensure the order of cell cycle events. Science 1989, 246:629-634.

4. Li R, Murray AW: Feedback control of mitosis in budding yeast. Cell 1991, 66:519-531.

5. Dobles M, Liberal V, Scott ML, Benezra R, Sorger PK: Chromosome missegregation and apoptosis in mice lacking the mitotic checkpoint protein Mad2. Cell 2000, 101:635-645.

6. Basto R, Brunk K, Vinogradova T, Peel N, Franz A, Khodjakov A, Raff JW: Centrosome amplification can initiate tumorigenesis in flies. Cell 2008, 133:1032-1042.

7. Clute P, Pines J: Temporal and spatial control of cyclin B1 destruction in metaphase. Nat Cell Biol 1999, 1:82-87.

8. Gascoigne KE, Taylor SS: Cancer cells display profound intra- and interline variation following prolonged exposure to antimitotic drugs. Cancer Cell 2008, 14:111-122.

9. Bakhoum SF, Thompson SL, Manning AL, Compton DA: Genome stability is ensured by temporal control of kinetochore-microtubule dynamics. Nat Cell Biol 2009, 11:27-35.

10. Yang Z, Kenny A, Brito D, Rieder CL: Cells satisfy the mitotic checkpoint in taxol and do so faster in concentrations that stabilize syntelic attachments. J Cell Biol 2009, 186:675684.

11. Rieder CL, Cole RW, Khodjakov A, Sluder G: The checkpoint delaying anaphase in response to chromosome monoorientation is mediated by an inhibitory signal produced by unattached kinetochores. J Cell Biol 1995, 130:941-948.

12. Pinsky BA, Biggins $S$ : The spindle checkpoint: tension versus attachment. Trends Cell Biol 2005, 15:486-493.

Published: 16 November 2009

doi:10.1186/jbiol195

(c) 2009 BioMed Central Ltd 